\title{
Apoptosis y liquen plano oral. Situación actual
}

\section{Apoptosis and oral lichen planus. Actual state}

\author{
Aguado Gil JM*, Rubio Flores D*
}

\section{RESUMEN}

El liquen plano oral (LPO) es una enfermedad mucocutánea inflamatoria crónica, con una etiología aún desconocida, de base autoinmune, que suele cursar con manifestaciones orales, con una clínica e histología características y de curso evolutivo benigno, pero susceptible de transformación maligna. En los últimos años se ha investigado la relación entre su patogenia y los mecanismos apoptóticos de destrucción celular.

Material y método: Búsqueda bibliográfica en el servidor de la U.S. National Library of Medicine and the National Institutes of Health (Pubmed) con las palabras clave "apoptosis AND oral lichen planus".

Discusión: Existen diferentes estudios que evalúan la relación de los diferentes marcadores apoptóticos (TNF$\alpha$, bcl-2, Fas, p53, BMP-4, granzima B, MMP...) con la patogenia, evolución, clínica y malignización del LPO. Para la determinación de estos factores se emplean técnicas de anatomía patológica e inmunohistoquímica (TUNEL, PCR,...).

Conclusión: no existe consenso en los resultados y las consiguientes conclusiones obtenidas en los diferentes estudios sobre la influencia de cada uno de los marcadores apoptóticos en el desarrollo de las lesiones de LPO. Es necesaria una mayor y más profunda investigación en búsqueda de un factor siempre asociado a las formas clínicas agresivas con mayor tendencia a la malignización.

Palabras clave: Liquen plano oral, apoptosis, p53, Bcl-2, queratinocitos.

\section{SUMMARY}

Oral lichen planus (OLP) is a mucocutaneous inflammatory chronic disease, with unknown etiology, autoinmune, usually associated with characteristical oral manifestations. Despite it has a benign evolution, is posible to become malign. Lately, relation between the pathogenesis and apoptotic cells destroy has been investigated. Methods: A bibliography survey was carried out with th U.S. National Library of Medicine and the National Institutes of Health (Pubmed) with the keywords "apoptosis AND oral lichen planus".

Discussion: Several trials evaluate the relationship among several apoptotic markers (TNF- $\alpha$, bcl-2, Fas, p53, BMP-4, granzyme B, MMP...) and OLP pathogenesis, evolution, clinic and malignization. These studies employed histology and immunohistochemistry (TUNEL, PCR)

Conclusion: Lack of consensus on results and conclusions about the influence of each apoptotic marker in the OLP development. Further investigation is required to obtain an apoptotic marker strongly associated with aggressive clinic and high-risk of malignancy.

Key words: Oral lichen planus, apoptosis, p53, Bcl-2, keratinocytes.

Fecha de recepción: Mayo 2008.

Aceptado para publicación: Mayo 2008.

* $\quad$ Licenciado en Odontología. Alumno del Máster en Ciencias Odontológicas (UCM). Curso 2007-2008.

Aguado Gil JM, Rubio Flores D. Apoptosis y liquen plano oral. Situación actual. Av. Odontoestomatol 2009; 25 (1): 11-18. 


\section{INTRODUCCIÓN}

Últimamente se están produciendo grandes avances en el diagnóstico y tratamiento del liquen plano oral, pero a día de hoy sigue siendo una entidad con muchos interrogantes tras de sí para todos los expertos en medicina oral, sobre todo en lo referente a su proceso de aparición y a su tratamiento. En este artículo se va a tratar su relación con la apoptosis, un tema que está siendo ampliamente estudiado en los últimos años.

El liquen plano oral (LPO) es una enfermedad mucocutánea inflamatoria crónica, con una etiología aún desconocida, de base autoinmune, que suele cursar con manifestaciones orales, con una clínica e histología características y de curso evolutivo benigno, pero susceptible de transformación maligna ${ }^{1}$.

Se considera la enfermedad no infecciosa más frecuente de la mucosa oral. Los autores cifran su incidencia entre un 0,2 y un $2 \%$ en la población general. Es más frecuente en mujeres (60-70\%), con un pico de prevalencia entre los 50 y 55 años. Según Bascones y cols, es casi exclusivo de la raza blanca ${ }^{2,3}$.

Pese a que, como se ha mencionado, la etiopatogenia del liquen plano es aún desconocida, se están estudiando mucho sus posibles relaciones con ciertas entidades y patologías. Está aceptada su asociación con niveles más elevados de ansiedad, mayor grado de depresión y un aumento de la vulnerabilidad a desórdenes psíquicos, especialmente las formas erosivas ${ }^{6}$. Se fundamenta en los niveles elevados de cortisol salival provocados por el estrés ${ }^{4}$. También se relaciona en una revisión sistemática de 25 estudios controlados, la proporción de sujetos con hepatitis $\mathrm{C}$, que era mayor en pacientes con LPO que en el grupo control, demostrando una diferencia estadísticamente significativa ${ }^{5}$.

En cuanto a la clínica, a nivel bucal el LPO se puede presentar de 3 formas diferentes ${ }^{6}$ :

- Reticular

- Eritematosa o atrófica

- Erosiva, ulcerada o bullosa

A nivel oral, se caracteriza por lesiones bilaterales más o menos simétricas con líneas blanquecinas (forma reticular) o lesiones atróficas (formas atrófica y erosiva) ${ }^{3}$, localizadas de forma más frecuente en la región posterior de la mucosa oral, lengua, encía, mucosa labial y bermellón del labio inferior. Aproximadamente un $10 \%$ de los pacientes presentan lesiones de tipo atrófico y erosivo limitadas a encía cursando con lo que se conoce como una gingivitis descamativa crónica ${ }^{7}$.

Aunque las lesiones suelen ser asintomáticas, las formas atrófico-erosivas cursan con sensación de quemazón, dolor, disfagia, dificultad en el habla... Para el tratamiento de la sintomatología asociada, se recomienda la eliminación de placa y cálculo y un adecuado mantenimiento de la higiene oral, la eliminación de posibles factores traumáticos y el empleo de corticosteroides, preferentemente en forma tópica, reservando los sistémicos para los casos más graves ${ }^{8}$.

En referencia a la histología, el LPO se caracteriza por ser una lesión con una intensa degeneración hidrópica vacuolar del citoplasma de las células basales- formando ampollas subepiteliales conocidas como "espacios de Max Joseph"-, un denso infiltrado inflamatorio celular difuso linfocitario en banda en contacto con la capa basal y la desaparición por sectores de la membrana basal Pas +. Está compuesto principalmente por linfocitos T (sobre todo CD4) y macrófagos. No hay relación entre la cantidad de linfocitos $\mathrm{T}$ y el tipo clínico y extensión de las lesiones. Aparecen también células de Langerhans, que se comportan como células presentadoras de antígenos para los linfocitos, induciendo lisis celular. Se ha demostrado la presencia de cuerpos hialinos esferoidales (cuerpos de Civatte), homogéneos, Pas + como consecuencia de la necrosis prematura de queratinocitos basales. En el epitelio aparecen hiperqueratosis y acantosis.

Aparecen también descritas en la literatura las conocidas como reacciones liquenoides, que son lesiones que parecen clínica e histológicamente liquen plano, pero que presentan una causa definible. Entre ellas podemos mencionar: materiales de obturación, autoinmune por trasplante de células hematopoyéticas y fármacos (antiinflamatorios no esteroideos [AINEs] e inhibidores de la encima de conversión de la angiotensina [IECAs]) ${ }^{7}$.

Uno de los principales problemas que hace que el LPO requiera de un seguimiento continuo del pa- 
ciente es su posibilidad de transformación maligna, que sigue siendo un tema de controversia entre los distintos autores. El potencial de malignización varía del 0,4 a más del $5 \%$ en periodos de revisión de 6 meses a 20 años, independientemente del tipo clínico y del tratamiento administrado ${ }^{6,7}$. La degeneración maligna es a carcinoma oral de células escamosas (COCE). Al no haberse localizado factores de riesgo claramente asociados, Gonzáles Moles y cols sugieren la implicación de alteraciones en los mecanismos de control del ciclo celular del epitelio oral con liquen plano $^{9}$. Parece que en pacientes con liquen plano con tumor primario hay un mayor riesgo (20-35,7\%) de desarrollo de múltiples carcinomas y sugiere la implicación del fenómeno de cancerización de campo.

Un término importante para este trabajo es el de apoptosis. Proviene del griego y significa "caída" (como las hojas de los árboles). Es el conjunto de procesos que se suceden en la muerte celular programada, caracterizado por una disminución de tamaño de las células, que luego se condensan y se fragmentan, liberándose cuerpos apoptóticos rodeados de membranas que son fagocitados por otras células ${ }^{10}$. Persigue la eliminación de células dañadas o inútiles, sin que se vean afectadas las células adyacentes. Se comporta como un eslabón de la cadena del ciclo celular ${ }^{11}$.

\section{MATERIAL Y MÉTODO}

Se ha realizado una búsqueda bibliográfica en el servidor de la U.S. National Library of Medicine and the National Institutes of Health (Pubmed), disponible en www.pubmed.gov. Se introducen como palabras clave "apoptosis AND oral lichen planus", limitando la búsqueda a los últimos 5 años. Aparecen 27 referencias, excluyendo aquéllas que no estuvieran en el idioma inglés o español. Así mismo se han consultados libros relacionados con el tema de la biblioteca de la Facultad de Odontología de la Universidad Complutense de Madrid.

\section{DISCUSIÓN}

La apoptosis, regulada por diversos genes, tiene un papel fundamental en la morfogénesis, homeostasis y regresión del cáncer. Esto adquiere una gran rele- vancia en relación a la tendencia a la malignización del LPO, aceptada a día de hoy con los estudios publicados $^{6,7,9,12}$.

Aún se desconocen los mecanismos por los cuales los linfocitos provocan apoptosis de los queratinocitos basales en el LPO aunque se han planteado tres posibles ejes que son capaces de activar la cascada de las caspasas provocando dicho fenómeno ${ }^{5}$ :

1. Secreción por las células T CD8 de TNF- $\alpha$ que se une por el receptor TNFR-1 al queratinocito basal, induciendo su apoptosis. Se justifica en base a niveles séricos aumentados de TNF- $\alpha$ y presencia de TNFR-1 en las capas basales y suprabasales del epitelio oral de las lesiones de LPO.

2. Superficie FAS-L del linfocito T CD 8 se une a la superficie FAS del queratinocito basal.

3. Secreción por células T CD 8 de granzima $B$, que penetra en el queratinocito a través de poros en la membrana inducidos por la porfirina. Kastelan y cols $^{13}$ (2004) demostraron la presencia de células positivas a porfirina en la proximidad de los queratinocitos apoptóticos, lo cual sugiere una posible relación con el daño celular.

En un estudio de Tobón-Arroyave y cols ${ }^{14}$ (2004) evaluaron los cambios estructurales y el papel de la caspasa- 3 asociados a la apoptosis de los queratinocitos en el LPO. En cuanto a los cambios estructurales, se demostraron niveles significativamente aumentados de células apoptóticas en LPO respecto al grupo control. Se observaron cuerpos apoptóticos redondeados, uniformes, con citoplasma eosinófilo y condensación de la cromatina nuclear en todos los cortes histológicos de LPO, en los estratos basal y espinoso, especialmente en las formas atróficas. En segundo lugar, determinaron una mayor expresión de caspasa- 3 a nivel de las células basales, lo que sugiere una activación de la cascada de las caspasas y sugiere una implicación de esta proteasa en la patogenia de la enfermedad.

Para González Moles y cols ${ }^{9}$ (2006) en su estudio con 51 pacientes con LPO y 26 controles, la apoptosis es poco frecuente o inexistente en el liquen plano, apoyado también por otros autores (Bascones y cols ${ }^{15}$ ), estando probablemente causada por un mecanismo 


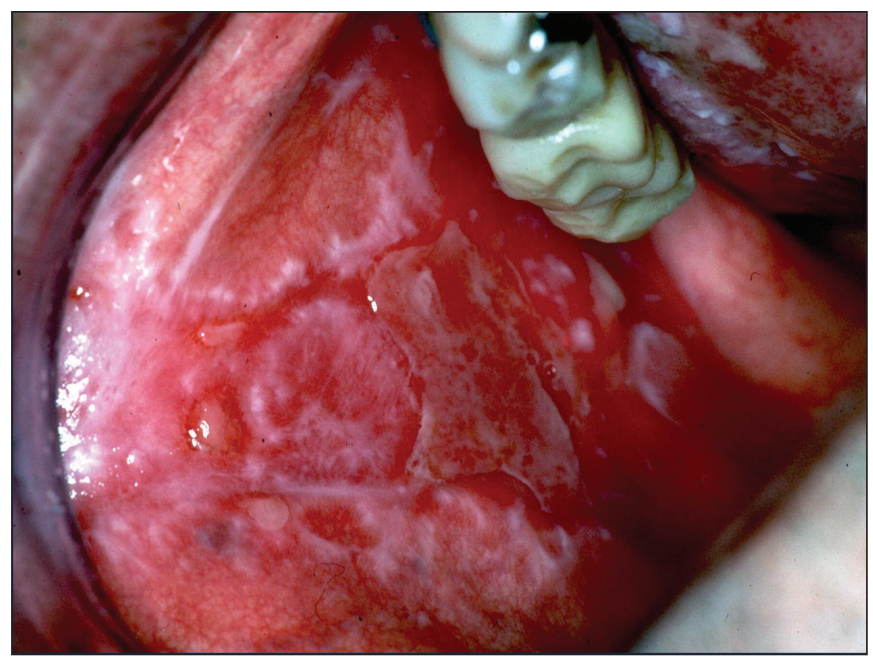

Fig. 1. Imagen clínica de una lesión de LPO atrófica en mucosa yugal (Dr. Antonio Bascones).

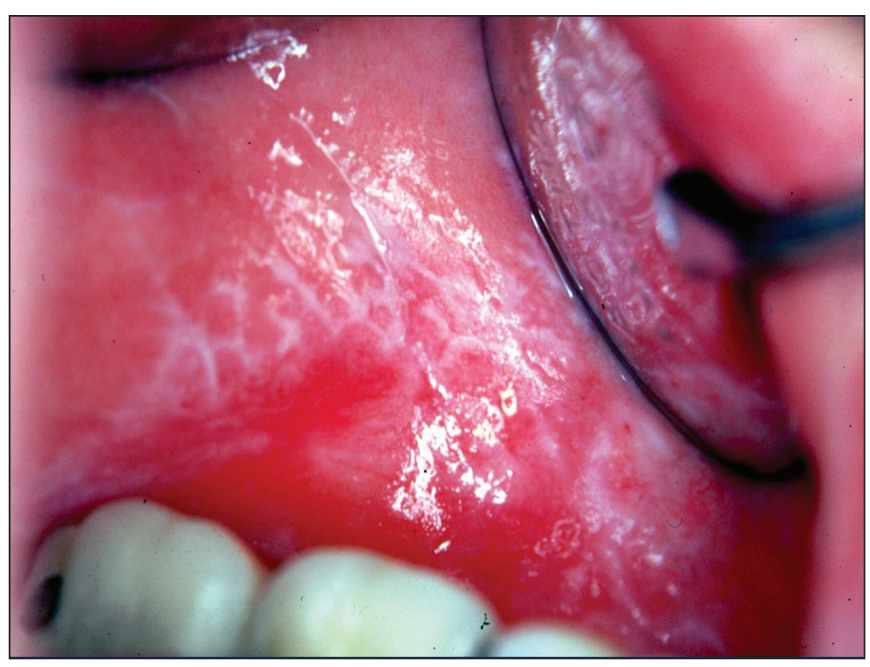

Fig. 2. Imagen clínica de lesión de LPO reticular en localización retrocomisural. (Dr. Antonio Bascones)

metaloproteinasas de matriz (MMP), relacionadas con los procesos necesarios para la remodelación tisular, la curación de heridas y la invasión tumoral como son la angiogénesis, la migración celular y la activación proteolítica de factores de crecimiento. La actividad de las MMP se regula por los inhibidores endógenos tisulares de metaloproteinasas (TIMP), implicados por tanto en procesos como la proliferación y apoptosis celular y procesos angiogénicos e inflamatorios. En su estudio compararon los niveles de estas proteinasas, por técnica de PCR, entre las formas erosivas con las reticulares así como pacientes sanos a modo de control. Los niveles de MMP-1 (colagenasa intersticial) y MMP-3 fueron significativamente mayores en la forma erosiva que en la reticular. Establecen como factor regulador de la evolución del LPO la ratio MMP-TIMP; así, los TIMP serían capaces de controlar la acción de las MMP en las formas reticulares, no así ya en las erosivas. Por lo tanto, establecen que el recuento de MMP (especialmente 1 y 3 ) puede ser un factor diagnóstico útil para las formas de LPO y su posible malignización.

Se ha planteado como posible manifestación de los procesos apoptóticos presentes en el LPO el adelgazamiento epitelial. Karatsaidis y cols ${ }^{18}$ (2003), en un estudio con 22 pacientes con LPO (10 con lesiones reticulares y 12 con lesiones eritematosas) y 11 pacientes sanos, no hallaron la presencia de apoptosis en las muestras de LPO, sugiriendo que el adelgaza- 
miento epitelial se debía más a una posible diferenciación terminal anormal o prematura de los queratinocitos o a una migración prematura de éstos a las capas superficiales. Para ellos, los cambios patológicos son debidos al infiltrado inflamatorio celular, que actúa a modo de señal para distintas citoquinas y factores de crecimiento (TNF- $\alpha$, IFN-g o IL-2) capaces de afectar el crecimiento y diferenciación celular epitelial.

Bascones- Ilundain y cols ${ }^{19}$ (2008) realizaron un análisis cuantitativo de dos mecanismos apoptóticos entre las formas atrófico- erosiva y reticular, la expresión de caspasa-3 (relacionada con la activación de otras caspasas y con la proteólisis) y Bax (perteneciente a la familia $\mathrm{Bcl}-2$ ). Se considera a la apoptosis como un mecanismo preventivo de transformación maligna de células epiteliales, con un mayor número de cuerpos de Civatte y exocitosis de linfocitos en la forma atrófico-erosiva que en la reticular, apoyando los nuevos hallazgos que contradicen el supuesto mayor riesgo de malignización de la forma atrófico- erosiva. En el estudio, no hallaron diferencias estadísticamente significativas para ninguno de los dos marcadores apoptóticos entre las dos formas, no pudiendo asociar la relación entre marcadores de apoptosis y detección precoz de riesgo de malignización.

En otro estudio del mismo grupo, evaluando como posible marcador apoptótico la degeneración por licuefacción mediante TUNEL e inmunohistoquímica para caspasa-3 y p21, concluyeron la ausencia de asociación inequívoca entre dicha degeneración, presente en la mayoría de casos, y marcadores apoptóticos (caspasa 3); sin embargo, sí hallaron asociación entre niveles elevados de p21 y una mayor licuefacción de la capa basal. ${ }^{20}$

Para este grupo investigador la persistencia de un intenso infiltrado inflamatorio, podría ser factor causal o predisponente a la malignización del LPO. Comprobaron las bajas tasas de apoptosis de los linfocitos $\mathrm{T}$, lo cual permite la persistencia del infiltrado. Éste puede provocar no sólo la inhibición de los mecanismos apoptóticos, sino también aumentar la tasa de proliferación de las células basales. ${ }^{21}$

Santoro y cols ${ }^{22}$ (2004) compararon los procesos apoptóticos entre las formas oral y cutánea del li- quen plano. Concluyen que en ambos el reclutamiento de células citotóxicas es mayor que en los controles sanos, siendo similar entre ellas. Sin embargo, sí encontraron diferencias en la expresión de granzimaB, Tia-1 y perforina en las células epiteliales y de granzima-B en las dérmicas, siendo mayor en el LPO que en la variante cutánea. La presencia de este mayor recuento de marcadores de apoptosis en localizaciones selectivas, sugiere su implicación en la patogenia del liquen plano.

Neppelberg y cols ${ }^{23}$ (2007) estudiaron la expresión en relación con la apoptosis celular de CD40, CD40 ligando, CD44 y cadherina epitelial. CD40 es una proteína transmembrana perteneciente a la superfamilia del receptor del factor de necrosis tumoral (TNFR), expresada por células con alto potencial proliferativo (queratinocitos, células $\mathrm{T}$, células dendríticas y macrófagos). El eje CD40-CD40 ligando juega un papel importante en la regulación del crecimiento celular, respuestas inflamatorias y apoptosis. La cadherina epitelial es una proteína de adhesión transmembrana relacionada con adhesión intercelular en epitelios escamosos estratificados. Su presencia previene la muerte celular por apoptosis. En tercer lugar, la proteína CD44 mantiene uniones intercelulares, así como el mantenimiento de la membrana basal. En los pacientes con LPO, los queratinocitos basales no expresan CD40 y se manifiesta una reducción en la expresión de cadherina epitelial especialmente en áreas de intenso infiltrado inflamatorio. No se encontraron diferencias para la expresión de CD 44 entre pacientes sanos y pacientes con LPO. La pérdida de cadherina epitelial contribuye a la destrucción de las células epiteliales facilitando la migración de células T al espacio epitelial.

En otra de las investigaciones de González- Moles y cols $^{24}$ (2007) se estudia la expresión de p53 y su papel en la patogenia del LPO, no encontrando asociación estadísticamente significativa entre los niveles de la proteína p53 y el marcador de apoptosis caspasa-3. Por ello, concluyen que la proteína p53 desempeña en el liquen plano un papel reparador del ADN, solo asociándose a malignización del LPO en caso de mutación del gen que la codifica.

En otros estudios, se han buscado marcadores apoptóticos a nivel sistémico. Así, Sklavounou- 
Andrikopoulou y cols ${ }^{25}$ (2004) evaluaron los niveles séricos de TNF- $\alpha$, Fas y Bcl- 2 en el LPO, encontrando niveles elevados de éstos en el grupo de pacientes con LPO. Los niveles elevados de TNF- $\alpha$ estarían relacionados con la inducción o perpetuación de procesos apoptóticos en el epitelio lesionado. No se han encontrado diferencias en los niveles de TNF- $\alpha$ entre las formas reticular y erosiva, no estando por tanto implicado el factor en el progreso del LPO. En cuanto al Fas, sus niveles séricos elevados sugieren el aumento en la unión entre queratinocitos basales y linfocitos T, promoviendo la activación de las caspasas. Por último, en referencia al Bcl-2, no se encontraron diferencias entre las formas erosivas respecto al grupo control, pero sí respecto a las formas reticulares. Estos menores niveles de Bcl-2 en las formas erosivas suscitan una mayor susceptibilidad a la apoptosis desde el infiltrado inflamatorio subepitelial. Es- tas diferencias entre las dos formas clínicas de LPO contrastan con los resultados obtenidos en los estudios de González- Moles ${ }^{9}$ y Bascones- Ilundain ${ }^{19}$.

Existen otros marcadores de apoptosis que pueden estar involucrados en la patogenia del LPO. Kim y cols $^{26}$ (2006) tomaron como referencia la proteína morfogénica ósea-4 (BMP-4). Esta proteína tiene un efecto estimulador de la diferenciación de células epiteliales, lo que parece que pueda estar relacionado con los procesos apoptóticos. Encontraron niveles elevados de BMP-4 en los pacientes con LPO respecto a los controles lo que parece inducir apoptosis por medio de p53 y MMP-1 y MMP-3.

El resumen de los resultados obtenidos en los diferentes estudios consultados se puede observar en la Tabla 1.

\section{TABLA 1: CUADRO ESQUEMÁTICO DE LOS RESULTADOS OBTENIDOS EN LOS ÚLTIMOS ESTUDIOS CLÍNICOS QUE RELACIONAN MARCADORES DE APOPTOSIS CON LPO}

\begin{tabular}{|c|c|}
\hline Estudio clínico & Resultados \\
\hline Tobón- Arroyave (2004) & - Aumento de caspasa 3. \\
\hline González- Moles (2006) & $\begin{array}{l}\text { - Apoptosis poco frecuente o inexistente. } \\
\text { - Aumento de p21. } \\
\text { - Bcl- } 2 \text { disminuida o ausente. }\end{array}$ \\
\hline Karatsaidis (2004) & - Aumento de núcleos TUNEL+ de linfocitos T CD4 y CD68. \\
\hline Mazzarella (2006) & - MMP-1 y 3 mayores en la forma erosiva respecto a la reticular. \\
\hline Karatsaidis (2003) & - Ausencia de apoptosis. \\
\hline Bascones- Ilundain (2008) & $\begin{array}{l}\text { - No diferencias en caspasa- } 3 \text { y Bcl- } 2 \text { entre las formas erosiva y } \\
\text { reticular de LPO. } \\
\text { - Persistencia de un intenso infiltrado inflamatorio sin apoptosis. } \\
\text { - Ausencia de asociación entre degeneración licuefactiva y marcado- } \\
\text { res apoptóticos. }\end{array}$ \\
\hline Santoro (2004) & $\begin{array}{l}\text { - Aumento de Granzima B, Tia-1 y perforina en LPO respecto a LP } \\
\text { cutáneo. }\end{array}$ \\
\hline Neppelberg (2007) & $\begin{array}{l}\text { - CD40, CD40L disminuidos. } \\
\text { - E- cadherina disminuida en la capa basal. } \\
\text { - CD44 igual que en sanos. }\end{array}$ \\
\hline González- Moles (2007) & - No hay diferencias de p53 entre LPO y sanos. \\
\hline Sklavounou- Andrikopoulou (2004) & $\begin{array}{l}\text { - Aumento de TNF- } \alpha \text { y Fas. } \\
\text { - Disminución de Bcl- } 2 \text { en formas erosivas respecto a reticulares. }\end{array}$ \\
\hline Kim (2006) & - Aumento de BMP-4. \\
\hline
\end{tabular}




\section{CONCLUSIONES}

1. No hay consenso entre los diferentes autores sobre si el LPO es una patología con predominio de fenómenos apoptóticos.

2. No existe acuerdo sobre el marcador apoptótico más frecuentemente relacionado con el LPO.

3. Muy probablemente el proceso apoptótico se deba a una asociación de los 3 ejes que activan la cascada de las caspasas.

4. Se precisan estudios a largo plazo con una amplia muestra monitorizando la mayoría de los marcadores apoptóticos, buscando diferencias significativas entre las distintas formas clínicas, la patogenia, la evolución y la malignización de los casos con LPO.

\section{BIBLIOGRAFÍA}

1. Blanco A. Liquen Plano Oral. Concepto. Pág, 15. Ed. Avances. Madrid. 2000.

2. Bascones A. Medicina Bucal. Capítulo 7. Pág. 124. Ed. Avances. $3^{a}$ edición. Madrid. 2004.

3. Bascones-llundain C, González Moles MA, Carrillo de Albornoz A, Bascones-Martínez A. Liquen plano oral (I). Aspectos clínicos, etiopatogénicos y epidemiológicos. Av. Odontoestomatol 2006; 22(1):11-9.

4. Koray M, Dulger O, Ak G, Horasanli S, Uçok A, Tanyeri $\mathrm{H}$ et al. The evaluation of anxiety and salivary cortisol levels in patients with oral lichen planus. Oral Dis 2003;9:298-301.

5. Lodi G, Scully C, Carrozzo M, Griffiths M, Sugerman P, Thongprasom K. current controversies in oral lichen planus: report of an international consensus meeting. Part 1 . Viral infections and etiopathogenesis. Oral Surg Oral Med Oral Pathol Oral Radiol Endod 2005;100: 40-51.

6. Eisen D, Carrozzo M, Bagan JV, Thongprasom K. Oral lichen planus: clinical features and management. Oral Dis 2005; 11: 338-49.
7. Scully C, Carrozzo M. Oral Mucosal Disease: Lichen Planus. Br J Oral Maxillofac Surg 2008; 46:15-21.

8. Lodi G, Scully C, Carrozzo M, Griffiths M, Sugerman P, Thongprasom K. current controversies in oral lichen planus: report of an international consensus meeting. Part 2: Clinical management and malignant transformation. Oral Surg Oral Med Oral Pathol Oral Radiol Endod 2005;100:164-78.

9. González-Moles MA, Bascones-Ilundáin C, Gil Montoya JA, Ruiz-Avila I, Delgado-Rodríguez M, Bascones-Martinez A. Cell cycle regulating mechanisms in oral lichen planus: molecular bases in epithelium predisposed to malignant transformation. Arch Oral Biol 2006;51:1093-1103.

10. Lodish H, Berk A, Zipursky SL, Matsudaira P, Baltimore D, Darnell JE. Biología celular y molecular. Pág. 1045. Editorial Medica Panamericana. $4^{\text {a }}$ edición. Madrid. 2002

11. Bascones-llundain C, González Moles MA, CampoTrapero J, Bascones-Martínez A. Liquen plano oral (II). Mecanismos apoptóticos y posible malignización. Av. Odontoestomatol 2006; 22 (1): 21-31.

12. Cheng B, Rhodus N, Williams B, Griffin R. Detection of apoptotic cells in whole saliva of patients with oral premalignant lesions: a preliminary study. Oral Surg Oral Med Oral Pathol Oral Radiol Endod 2004;97:465-70.

13. Kastelan M, Massari LP, Gruber F, Zamolo G, Saura G, Coklo M, Rukavina D. The role of perforin-mediated apoptosis in lichen planus lesions. Arch Dermatol Res 2004;296:226-30

14. Tobón-Arroyave SI, Villegas-Acosta FA, RuizRestrepo SM, Vieco-Durán B, Restrepo-Misas M, Londoño-López ML. Expresión of caspase-3 and structural changes associated with apoptotic cell deth of keratinocytes in oral lichen planus. Oral Dis 2004;10:173-8.

15. Bascones C, Gonzalez-Moles MA, Esparza G, Bravo M, Acevedo A, Gil-Montoya JA, Bascones 
A. Apoptosis and cell cycle arrest in oral lichen planus Hypothesis on their possible influence on its malignant transformation. Arch Oral Biol. 2005 Oct;50(10):873-81.

16. Karatsaidis A, Schreurs O, Axell, T, Helgeland K, Schenck K. Identity of TUNEL-positive cells in the oral buccal epithelium of normal mucosa and lichen lesions. J Oral Pathol Med 2004;33:264-8.

17. Mazzarella N, Femiano F, Gombos F, De Rosa A, Giuliano M. Matrix metalloproteinase gene expression in oral lichen planus: erosive vs. Reticular forms. J Eur Acad Dermatol Venereol 2006; 20:953-7.

18. Karatsaidis A, Schreurs O, Helgeland K, Axell T, Schenck K. Erythematous and reticular forms of oral lichen planus and oral lichenoid reactions differ in pathological features related to disease activity. J Oral Pathol Med 2003;32:275-81.

19. Bascones-Ilundain C, González-Moles MA, Campo-Trapero J, Gil-Montoya JA, Esparza-Gómez GC, Cano-Sánchez $J$ et al. No differences in caspase-3 and Bax expresión in atrophic-erosive vs. Reticular oral lichen planus. J Eur Acad Dermatol Venereol 2008;22:204-12.

20. Bascones-Ilundain C, Gonzalez-Moles MA, Esparza G, Gil-Montoya JA, Bascones-Martinez A. Significance of liquefaction degeneration in oral lichen planus: a study of its relationship with apoptosis and cell cycle arrest markers. Clin Exp Dermatol 2007;32:556-63.
21.Bascones-Ilundain C, Gonzalez-Moles MA, Esparza-Gómez G, Gil-Montoya JA, Bascones-Martínez A. Importance of apoptotic mechanisms in inflammatory infiltrate of oral lichen planus lesions. Anticancer Res 2006; 26 (1A):357-62.

22. Santoro A, Majorana A, Bardellini E, Gentili F, Festa S, Sapelli P et al. Cytotoxic molecule expresión and epithelial cell apoptosis in oral and cutaneous lichen planus. Am J Clin Pathol 2004; 121:758-64.

23. Neppelberg E, Loro LL, Oijordsbakken G, Johannessen AC. Altered CD40 and E-cadherin expression-putative role in oral lichen planus. $\mathrm{J}$ Oral Pathol Med 2007;36:153-60.

24. González-Moles MA, Gil-Montoya JA, Ruiz-Ávila I, Esteban F, Bascones-Martínez A. Differences in the expresión of p53 protein in oral lichen planus based on the use of monoclonal antobodies D07 and pAB 240. Oral Oncol [serial on the Internet] 2007.06.013. Available from doi:10.1016/j. oraloncology.2007.06.013.

25. Sklavounou-Andrikopoulou A, Chrysomali E, Iakovou M, Garinis GA, Karameris A. Elevated serum levels of the apoptosis related molecules TNF- $\alpha$, Fas/Apo- 1 and Bcl-2 in oral lichen planus. J Oral Pathol Med 2004;33:386-90.

26. Kim SG, Chae CH, Cho BO, Kim HN, Kim HJ, Kim IS et al. Apoptosis of oral epithelial cells in oral lichen planus caused by upregulation of BMP4. J Oral Pathol Med 2006;35:37-45. 